\title{
(C) OPEN ACCESS \\ On average, a professional rugby union player is more likely than not to sustain a concussion after 25 matches
}

\author{
James Rafferty, ${ }_{1}$ Craig Ranson, ${ }^{2}$ Giles Oatley ${ }_{1}{ }^{3}$ Mohamed Mostafa, ${ }_{1}{ }^{4}$ \\ Prabhat Mathema, ${ }^{5}$ Tom Crick, ${ }^{\oplus 6}$ Isabel S Moore ${ }^{\oplus}$
}

${ }^{1}$ Medical School, Swansea University, Swansea, UK ${ }^{2}$ English Institute of Sport, Manchester, UK

${ }^{3}$ School of Engineering and Information Technology, Murdoch University, Perth, Western Australia, Australia ${ }^{4}$ Social Data Science Lab, School of Computer Science and Informatics, Cardiff University, Cardiff, UK

${ }^{5}$ Welsh Rugby Union, Cardiff, UK ${ }^{6}$ Department of Computing and Information Systems, Cardiff Metropolitan University, Cardiff, UK

${ }^{7}$ Cardiff School of Sport and Health Sciences, Cardiff Metropolitan University, Cardiff, UK

\section{Correspondence to} Dr Isabel S Moore, Cardiff School of Sport and Health Sciences, Cardiff Metropolitan University, Cardiff CF23 6XD, UK; imoore@cardiffmet.ac.uk

Accepted 17 February 2018 Published Online First 12 March 2018

\begin{abstract}
Objectives To investigate concussion injury rates, the likelihood of sustaining concussion relative to the number of rugby union matches and the risk of subsequent injury following concussion.

Methods A four-season (2012/2013-2015/2016) prospective cohort study of injuries in professional level (club and international) rugby union. Incidence (injuries/1000 player-match-hours), severity (days lost per injury) and number of professional matches conferring a large risk of concussion were determined. The risk of injury following concussion was assessed using a survival model.
\end{abstract}

Results Concussion incidence increased from 7.9 (95\% CI 5.1 to 11.7$)$ to 21.5 injuries/1000 player-matchhours $(95 \% \mathrm{Cl} 16.4$ to 27.6$)$ over the four seasons for combined club and international rugby union. Concussion severity was unchanged over time (median: 9 days). Players were at a greater risk of sustaining a concussion than not after an exposure of 25 matches (95\% Cl 19 to 32). Injury risk (any injury) was 38\% greater (HR 1.38; $95 \% \mathrm{Cl} 1.21$ to 1.56) following concussion than after a non-concussive injury. Injuries to the head and neck (HR 1.34; 95\% Cl 1.06 to 1.70), upper limb (HR 1.59; $95 \% \mathrm{Cl} 1.19$ to 2.12 ), pelvic region (HR 2.07; 95\% Cl 1.18 to 3.65) and the lower limb (HR $1.60 ; 95 \% \mathrm{Cl} 1.21$ to 2.10 ) were more likely following concussion than after a non-concussive injury.

Conclusion Concussion incidence increased, while severity remained unchanged, during the 4 years of this study. Playing more than 25 matches in the 2015/2016 season meant that sustaining concussion was more likely than not sustaining concussion. The $38 \%$ greater injury risk after concussive injury (compared with nonconcussive injury) suggests return to play protocols warrant investigation.

\section{INTRODUCTION}

A high proportion of professional rugby union players sustain multiple injuries over consecutive seasons. ${ }^{1}$ Of the common injuries, concussion has the highest match injury incidence, with the English Rugby Football Union (RFU) reporting a rate of $15.8 / 1000$ player-match-hours in $2015 / 2016 .^{2}$ This contrasts with earlier injury surveillance in 2006 reporting concussion incidence to be $1.4 / 1000$ player-match-hours within South African professional rugby union, ${ }^{3}$ and a mean incidence of 4.7/1000 player-match-hours cited in a 2014 worldwide rugby union injury meta-analysis. ${ }^{4}$ However, the low injury incidence in earlier years may represent under-reporting, meaning recent injury data from 2012 onwards could provide better estimates of true concussion incidence due to the roll-out of the Head Injury Assessment and concussion education programmes. $^{5}$

At the professional level, injury risk also appears to vary between club and international rugby union, with international match incidence being twice that of club rugby. ${ }^{6}$ Yet, no study has directly compared injury rates that include data from players who are involved with both the international and club levels, while also ensuring similar injury surveillance methodology. Additionally, recent RFU injury data indicate that concussion incidence has been increasing at club level over recent years, but injury severity appears to have remained relatively unchanged. ${ }^{2}$ However, this longitudinal data source has not yet been assessed at the international level.

Concussion was associated with $60 \%$ risk of subsequent injury (of any type) in rugby union ${ }^{7}$ and $50 \%$ in association football (soccer) ${ }^{8}$ in addition to a greater risk of sustaining lower limb injuries in college-level contact sports. ${ }^{10}$ Subsequent injuries also occur more quickly following a concussion than a non-concussive injury. ${ }^{7}$ However, it is not known what tissue types and body areas are at increased risk in rugby union.

The aims of this study were to: (1) determine whether the incidence and severity of concussion have changed over the surveillance period in club and international rugby union; (2) quantify the likelihood of a player sustaining concussion relative to the number of rugby union matches; (3) establish whether concussion is associated with an increased risk of subsequent injury in professional rugby union; and (4) examine if concussion confers greater risk of specific types of subsequent injury.

\section{METHODS}

Data collection

The Welsh Rugby Union injury surveillance programme has established an injury database collected from the Wales senior men's international team and the four professional Welsh rugby clubs (Cardiff Blues, Dragons, Ospreys and Scarlets) for the 4 years spanning the 2012/2013-2015/2016 seasons. Ethics approval was obtained from the Cardiff Metropolitan University School of Sport Ethics Committee and informed consent was obtained from players involved in the first team squads each season. The teams' physiotherapist recorded all time loss injuries during each season 
and submitted injury records at the end of each month, or end of each tournament in the case of the international team, to Cardiff Metropolitan University (ISM). Any discrepancies in injury records were immediately checked and reconfirmed following the submission of the data to the independent researcher. For the purpose of this study, only injuries sustained during matches were used for calculations of incidence, severity and subsequent injury risk, with all other time loss presented to provide a contextual understanding.

The injury definition and data collection procedures complied with the international consensus of injury surveillance in rugby union. ${ }^{11}$ Injuries were coded using the Orchard Sports Injury Classification System V.10. ${ }^{12}$ Additionally, each injury included information about the activity at time of injury (eg, match/ training, contact/non-contact), mode of onset and number of days lost per injury. Exposure was calculated by assuming each match lasted $80 \mathrm{~min}$ and each team consisted of 15 players. Occurrences where players were withdrawn due to injury or foul play (yellow/red cards) leaving fewer than 15 players per team in play were not accounted for.

\section{Incidence and severity}

Injury frequency for concussion sustained during matches was characterised by injury incidence, which is the mean number of concussions sustained in 1000 player-match-hours. Incidences were calculated for club and international teams separately, and collectively by combining the club and international injuries and exposure. To compare two injury incidences a rate ratio (RR) was calculated. The $95 \%$ confidence interval (CI) was calculated using normal distribution, since the log of the ratio of two incidence rates is normal. Two incidences were significantly different if the $95 \% \mathrm{CI}$ for the RR did not intersect with unity.

Injury severity refers to the number of days lost of training and match play due to an injury. The distribution of injury days lost was found to be skewed and did not fit well to any probability distribution so the median was used to represent days lost. The positive (right) skewness originates from most injuries resolving in a relatively short time, with a small number of injuries requiring longer recoveries. Uncertainties for injury severity were calculated using bootstrapping. ${ }^{13}$ Comparison of severity distributions was done using a Kolmogorov-Smirnov two-sample test. A weighted linear least squares fit was used to determine whether incidence and severity changed over time. Weights for the fit were taken to be the reciprocal of the size of the $95 \% \mathrm{CI}$, meaning points with narrower CIs were weighted more highly.

\section{Injury risk}

The standard metric used to report injury incidence is the number of injuries per 1000 player-match-hours. This metric allows comparisons to be made across different sporting populations, but it is difficult to infer specific player risk. Therefore, the incidence was used to calculate the risk of injury to a single player. The metric used to quantify the risk was the number of matches required for an injury to be more likely than not. The formula used to compute this quantity was:

$$
P(n)=e^{-n \lambda_{1}}
$$

where $n$ is the number of player matches and $\lambda_{1}$ is the incidence of an injury per player match, that is, 80 player-minutes of exposure (see online supplementary 1 for the derivation of this formula). The smallest $n$ such that $P(n)<0.5$ is the number of matches where there is a higher probability than not of an injury being sustained by a single player. This metric represents the average risk over the whole team, and does not take into account differences between players. Club and international injuries were combined to avoid undercounting concussion injuries. As such the metric will be referred to as the risk to the average player. The Python programming language (V.2.7.12) with numpy (V.1.11.2), scipy (V.0.18.1), statsmodels (V.0.6.1) and matplotlib (V.1.5.3) libraries was used for incidence, severity and risk calculations. ${ }^{14}$

\section{Subsequent injury risk}

The Andersen-Gill extension to the Cox proportional hazards model was used to quantify the risk of subsequent injury following return to play. ${ }^{15} 16$ HRs were calculated to compare players who had suffered concussions with those who had not suffered a concussion. Several preprocessing steps were taken to prepare the data. First, only active players on team rosters were selected to ensure each player had a complete set of injuries; if a player was not on a team roster for a Welsh club the injury was excluded. Club and international injuries were combined to avoid undercounting injuries to players who represented the national team.

Following this step, players were sorted into those who sustained at least one concussion and those who had not. The injury data for players who had suffered no concussions formed the non-concussive injury set used for reference comparisons. Players who had suffered a concussion had their injury data split into two sets: (1) injuries sustained prior to the first concussion and (2) injuries sustained following the first concussion. For the non-concussive injury set a 'first' injury was randomly chosen from each player's injury data to allow a similar split to be determined. The longest possible interval between injuries was 4 years because no time frame limit was imposed. Models were evaluated using the log-likelihood test. A p value greater than 0.05 indicated that the null hypothesis should be assumed and all HRs were consistent with unity. The $\mathrm{R}$ programming language (V.3.3.1) and the survival library (V.2.40-1) were used to perform the survival analysis. ${ }^{1718}$

\section{RESULTS}

\section{Incidence, severity and risk}

The data set contained 2441 injuries to 367 players, from a total player pool of 429 players. Therefore, 86\% of players sustained an injury during the surveillance period. Match injuries accounted for 1602 injuries, while there were 514 injuries during training. The remaining 325 injuries were sustained during other activities. The total match exposure was 11960 player-match-hours, of which 1000 player-match-hours were from international matches.

The match injury incidence over the surveillance period was 94.5 injuries/1000 player-match-hours (95\% CI 89.1 to 100.2). There was a lower match injury incidence in club rugby (87.0 injuries/1000 player-match-hours; 95\% CI 81.5 to 92.7 ) compared with international rugby (177.0 injuries/1000 player-match-hours; 95\% CI 151.9 to 205.1; RR 2.04; 95\% CI 1.73 to 2.39 ). The overall median severity for all injuries was 10 days lost (95\% CI 9 to 10). There was no difference between the median severity for club rugby (10 days lost; $95 \%$ CI 10 to 11) and international rugby (9 days lost; $95 \%$ CI 8 to 10 ).

Concussion was the most common injury $(10 \%$ of club and international injuries combined) and the incidence was shown to increase over the four seasons of club, and club and international rugby combined (table 1 ; figure 1 ). The severity of concussion 
Table 1 The match injury incidence (injuries per 1000 player-match-hours) of concussion in the first and last seasons of the injury surveillance period, and the proportionality constant from a weighted least squares fit of the incidences from all seasons

\begin{tabular}{|c|c|c|c|}
\hline Data set & Incidence 2012-2013 & Incidence 2015-2016 & $\begin{array}{l}\text { Weighted least squares proportionality } \\
\text { constant }\end{array}$ \\
\hline Club rugby & $7.1(4.3,11.0)$ & $21.4(16.0,28.0)$ & $4.94(1.69,8.19)$ \\
\hline International rugby & $18.2(5.0,46.6)$ & $22.2(9.6,43.8)$ & $1.99(-12.69,16.67)$ \\
\hline Combined club and international rugby & $7.9(5.1,11.7)$ & $21.5(16.4,27.6)$ & $4.68(0.81,8.55)$ \\
\hline
\end{tabular}

Rows in bold indicate where the proportionality constant significantly differs from zero.

did not change over time in club, international, and club and international rugby combined. The median severity of concussion over the time period was the same for club, international, and club and international rugby combined (9 days; 95\% CI 8 to 9). The average player had a higher risk of sustaining a concussion than not after 25 matches (95\% CI 19 to 33 ) in club rugby, after 24 matches (95\% CI 12 to 55 ) in international rugby and after 25 matches (95\% CI 19 to 32) in club and international rugby combined, based on the incidence from the 2015-2016 season. Considering the squad size and player-specific match exposure per season across the 4 years, the proportion of the squad playing more than 25 matches per season was $10 \%$.

\section{Subsequent injury risk}

There was a greater subsequent injury risk following a concussion compared to following a non-concussive injury (HR 1.38; 95\% CI 1.21 to 1.56 ), with players who sustained a concussion having a $38 \%$ greater injury risk than players who did not sustain a concussion. Injury risk was assessed before and after players had sustained a concussion, with players having a $23 \%$ greater injury risk after concussion than before concussion (HR 1.23 ; $95 \%$ CI 1.05 to 1.44 ). No difference in injury risk was found between players prior to sustaining a concussion and players prior to sustaining a non-concussive injury (HR 1.13; $95 \%$ CI 0.97 to 1.31 ). The median time to next injury following a concussion was 36 days (95\% CI 32 to 40), which was shorter than the time to next injury following an injury other than a concussion (49 days; 95\% CI 44 to 53 ).

Risk of injuries to specific body regions was greater after concussion than prior to concussion (table 2). Injuries to the head and neck (HR 1.34; 95\% CI 1.06 to 1.70), upper limb (shoulder and arm, HR 1.59; 95\% CI 1.19 to 2.12 ), pelvic region (buttock and groin, HR 2.07; 95\% CI 1.18 to 3.65 ) and

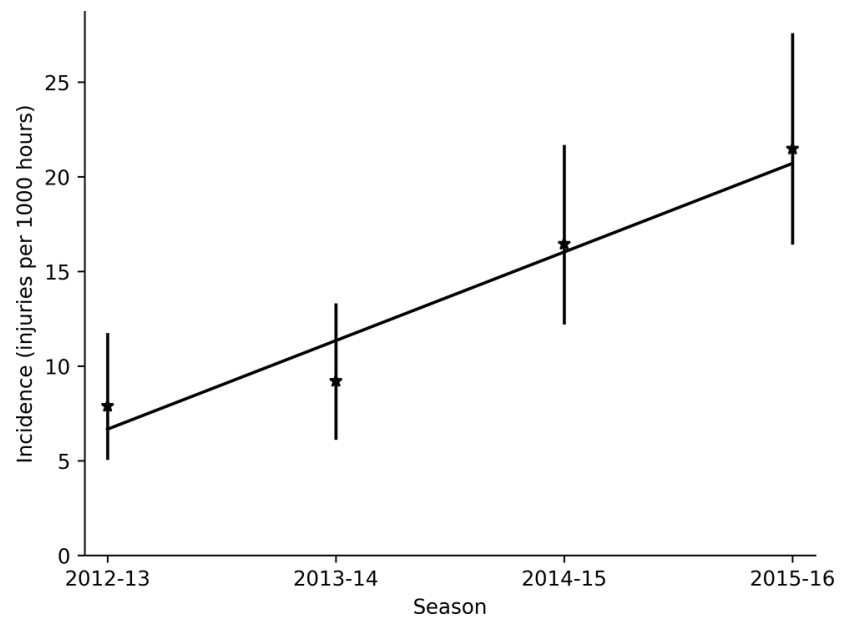

Figure 1 The match injury incidence $(95 \% \mathrm{Cl})$ of concussion in club and international rugby combined across the four seasons. the lower limb (leg, ankle and foot, HR 1.60; 95\% CI 1.21 to 2.10) were more likely in players following a concussion than to players who did not suffer a concussion. Injuries to joints and ligaments (HR 1.49; 95\% CI 1.22 to 1.81 ) and to muscles and tendons (HR $1.38 ; 95 \%$ CI 1.12 to 1.70 ) were also more likely following a concussion (table 3 ).

\section{DISCUSSION}

In Welsh Rugby Union, concussion injury incidence increased over the four seasons at a club level, but not international level; severity remained unchanged at both levels. Players were more likely than not to sustain a concussion after 25 matches and there was a $38 \%$ greater injury risk after concussion compared to following a non-concussive injury. Specifically, the subsequent injury risk was greater for the head and neck, upper limb, pelvic region and lower limb.

\section{Change in incidence and severity of concussion}

Our finding of an increasing secular trend in concussion incidence in elite Welsh Rugby Union, when combined with findings from the RFU Injury Surveillance Project, ${ }^{2}$ suggests that concussion incidence is rising throughout the professional game. At the international level, the concussion incidence was higher than in previous reports. ${ }^{619-21}$ However, the low level of exposure for the international team led to larger uncertainties. Combining longitudinal injury surveillance data from multiple international teams is recommended to see whether the recent increase in concussion persists at the international level.

World Rugby, rugby union's international governing body, has implemented several concussion policies at the professional level. Others have speculated that the Head Injury Assessment protocol led to a rise in the reported incidence of concussion following its introduction as a pilot in 2012 and officially in $2014 .^{2}$ As a result, in the $2014 / 2015$ season, concussions may have been reported that previously may not have been included. However, the continued rise in incidence in the 2015/2016 season implies there are additional factors other than stakeholder awareness that contribute to the increased concussion incidence. Identifying concussion risk factors is an important next step. Researchers may need to take into account surface-specific

Table 2 Hazard Ratios (HR) between injuries following a concussion versus following a non-concussion injury split up by body region

\begin{tabular}{lll}
\hline Body area & HR $(95 \% \mathrm{Cl})$ & Fit p value \\
\hline Head and neck & $1.34(1.06$ to 1.70$)$ & 0.028 \\
\hline Upper limb (arm and shoulder) & $1.59(1.19$ to 2.12$)$ & 0.009 \\
Abdomen, chest and back & $1.42(0.80$ to 2.55$)$ & 0.021 \\
\hline Pelvic region (buttock and groin) & $2.07(1.18$ to 3.65$)$ & 0.034 \\
Knee and upper leg & $1.12(0.90$ to 1.39$)$ & 0.636 \\
\hline Lower limb (ankle, foot and lower leg) & $1.60(1.21$ to 2.10$)$ & 0.006 \\
\hline
\end{tabular}

Rows in bold indicate both a significant HR and that the model fit was significant to $95 \%$ confidence. 


\begin{tabular}{lll}
\hline $\begin{array}{l}\text { Table } 3 \\
\text { versus following a non-concussion injury split up by tissue type }\end{array}$ \\
\hline Tissue type & HR $(95 \% \mathrm{Cl})$ & Fit p value \\
\hline Bone & $1.22(0.76$ to 1.97$)$ & 0.668 \\
\hline Joint and ligament & $1.49(1.22$ to 1.81$)$ & 0.002 \\
Muscle and tendon & $1.38(1.12$ to 1.70$)$ & 0.006 \\
Skin & $1.69(0.62$ to 4.60$)$ & 0.387 \\
Brain & $1.32(0.88$ to 1.99$)$ & 0.307 \\
Other & $1.49(1.05$ to 2.10$)$ & 0.071 \\
\hline
\end{tabular}

Rows in bold indicate both a significant HR and that the model fit was significant to $95 \%$ confidence.

match play demands (eg, tackles made and tackle technique) as grass has a greater concussion incidence than artificial surface. ${ }^{22}$ Furthermore, $50 \%$ of concussions occur in the tackle event ${ }^{7}$ and recent reports show that an upright posture by the tackler increases the risk of the tackler needing a Head Injury Assessment compared with a bent at the waist posture. ${ }^{23}$ Note that the latter technique is outlined as best practice in World Rugby's coaching education. ${ }^{24}$ Therefore, assessing whether tackling behaviour has changed over recent years may provide further insight into changes in concussion incidence.

\section{Concussion risk}

The average rugby union player had a higher risk of sustaining a concussion than not after 25 matches; this rate was three times higher than the next most frequent injury in our data (thigh haematoma). Additionally, based on current season lengths (mean 34 matches) the data show that 1 in 10 players will surpass the 25-match threshold of concussion risk each season. However, with concussion incidence rising year on year and 17\% of professional rugby union players reporting a concussion in the 2015-2016 season, ${ }^{2}$ this appears to be a conservative estimate possibly due to being an 'average' player metric. Nevertheless, the data suggest that longer seasons would potentially expose more players to the risk of concussion due to a greater proportion of the squad playing more than 25 matches each season, meaning decisions regarding season length should consider the injury risk implications.

\section{Subsequent injury risk}

The subsequent injury risk to players following a concussion was $38 \%$ higher than for players who did not sustain a concussion. Although the increased risk was significant, it was lower than the $60 \%$ reported for similar level players in the RFU Premier$\operatorname{ship}^{7}$ and lower than the $47 \%$ reported in professional association football. ${ }^{8}$ When considering studies in rugby union, the reported risks are consistent with each other, within calculated errors, with the combined estimate of subsequent injury risk in rugby union from this study and Cross et al's ${ }^{7}$ study being 1.49 (95\% CI 1.37 to 1.61). When the association football (soccer) data are included, ${ }^{8}$ the combined estimate of subsequent injury risk is $48 \%$ (HR $1.48 ; 95 \% \mathrm{CI} 1.30$ to 1.67 ). It appears that the increased injury risk (all injuries) following concussion is not specific to a single jurisdiction or sport.

The mechanisms underpinning this increased injury risk are not known. However, with the consistency between the two rugby union studies it could be speculated that the current length of the graded return to play protocol is insufficient because players can go from complete rest to full match play within 6 days. ${ }^{25}$ Previous research has shown that increases in weekly workload that are greater than $10 \%$ were associated with an increased injury risk. ${ }^{26}$ Consequently, if a longer graded return to play protocol was implemented, workloads could be progressed gradually to safer levels, which may mitigate some of the subsequent injury risk by ensuring 'spikes ${ }^{27}$ in acute workloads are avoided.

There were several body regions, in addition to joint, ligament, muscle and tendon injuries, that had a greater injury risk after concussion. A greater lower extremity injury risk was reported in collegiate athletes from multiple sports. ${ }^{9} 10$ Possible underlying mechanisms include altered balance strategies ${ }^{28}$ and impaired dynamic stability, ${ }^{29}{ }^{30}$ in particular dynamic balance ${ }^{28}$ and walking gait, ${ }^{29} 30$ which are negatively affected following concussion. This may also explain part of the increased injury risk to the joint, ligament, muscle and tendon structures, as loading across these structures may differ following concussion. We contend that small changes to balance and gait after concussion may increase subsequent injury risk. ${ }^{7}$

This is the first study to identify the head and neck, and upper extremity body regions to also have an increased risk following concussion. It is conceivable that the nature of contact in rugby union exposes players to a heightened risk to these body areas, with the tackle event a common injury mechanism. ${ }^{26}$ Additionally, if players are tackling higher up the body (eg, shoulder and above), a Head Injury Assessment is four times as likely than if tackling lower down the body. ${ }^{23}$ Therefore, the high initial risk of concussion and subsequent risk to upper body areas may be a by-product of tackle technique that does not follow recommended guidelines. ${ }^{24}$ However, contact is unavoidable in rugby union and it is unlikely that all concussions are due to improper tackle technique. As a result, further mechanistic work is required to understand whether the increased risk to these body areas is preventable within rugby union by considering whether factors such as oculomotor and vestibular dysfunction, ${ }^{31}$ and impaired dynamic stability and proprioception ${ }^{29} 30$ affect tackle technique. The combination of epidemiological and experimental data supports the recent concussion consensus statement ${ }^{32}$ that graduated return to play protocols should look to include multifaceted strategies such as neuromuscular control, and oculomotor and vestibular protocols to both aid identification and quantification of postconcussion symptoms, and mitigate subsequent injury risk.

\section{Limitations and strengths}

The small number of teams involved in the study is a limitation. However, the injury data were checked every month of every season, minimising the number of incomplete injury records. Additionally, by tracking international players from clubs to the national team, we have provided the first subsequent injury analysis in professional sport that has included all time loss injuries sustained by a player, regardless of when it occurred. It should also be noted that the probability metric for injury incidence is the mean probability over the whole team. Variations among individual players are ignored, which limits the conclusions that can be drawn for individuals.

\section{CONCLUSION}

This is the first study to quantify the player risk of sustaining a concussion using a metric that estimates the number of matches a player can participate in before the risk of concussion passes the 0.5 level. On average, rugby union players were more likely than not to sustain concussion after 25 matches. We have confirmed previous reports that there is an increased subsequent injury risk after concussion, and estimate the combined HR from this study and two others ${ }^{67}$ is 1.48 (95\% CI 1.30 to 1.67 ). Following concussion, several body regions were at greater risk of injury, 
specifically the head and neck, shoulder and arm, buttock and groin, and the lower limb (leg, ankle and foot). The types of injuries with greater risk were joint and ligament, and muscle and tendon. The mechanisms that underpin these concussion-associated risks warrant investigation.

What are the findings?

- Incidence of concussion has increased year on year during the study.

- Players are more likely than not to sustain a concussion after 25 matches.

- On average, $10 \%$ of squad players were exposed to more than 25 matches per season.

- Across football codes (association football and rugby union) the subsequent injury risk after concussion is $48 \%$ higher than for players who have not sustained a concussion.

\section{How might it impact on clinical practice in the future?}

- Our study reinforces previous studies showing a greater risk of subsequent injury after concussion (compared with players who have not suffered concussion) and a secular increase in concussion incidence. This has now been demonstrated in several sports at different competitive levels.

- Monitoring and potentially limiting the number of matches players are involved in during a season to 25 matches is recommended.

- Multiple body areas and structures have greater injury risk following concussion. Multifaceted strategies during graduated return to play may aid identification of postconcussion symptoms or mitigate subsequent injury risk.

- Whether longer concussion rehabilitation periods would mitigate the increased rates of subsequent injuries remains to be tested.

Acknowledgements The authors thank the team physiotherapists and strength and conditioning coaches who diligently recorded all the injury and exposure data, and Professor Stephen Mellalieu (Cardiff Metropolitan University) for helpful comments on an initial draft.

Contributors ISM, GO and CR conceived and designed the study. JR analysed and interpreted the data. ISM and JR prepared the first draft of the manuscript. JR, ISM, $C R, G O, T C, M M$ and $P M$ all made substantial contributions to the revision of the manuscript prior to submission.

Funding World Rugby and Welsh Rugby Union.

Competing interests ISM and CR have received a research grant from Welsh Rugby Union. ISM, CR, GO, MM and TC have received a research grant from World Rugby. PM is head of Medical Services at Welsh Rugby Union and CR was a physiotherapist for Welsh Rugby Union during the conduct of the study.

Patient consent Detail has been removed from this case description/these case descriptions to ensure anonymity. The editors and reviewers have seen the detailed information available and are satisfied that the information backs up the case the authors are making.

Ethics approval Cardiff Metropolitan University School of Sport Ethics Committee.

Provenance and peer review Not commissioned; externally peer reviewed.

Open access This is an open access article distributed in accordance with the Creative Commons Attribution Non Commercial (CC BY-NC 4.0) license, which permits others to distribute, remix, adapt, build upon this work non-commercially, and license their derivative works on different terms, provided the original work is properly cited and the use is non-commercial. See: http://creativecommons.org/ licenses/by-nc/4.0/

(c) Article author(s) (or their employer(s) unless otherwise stated in the text of the article) 2019. All rights reserved. No commercial use is permitted unless otherwise expressly granted.

\section{REFERENCES}

1 Moore IS, Mount S, Mathema P, et al. Application of the subsequent injury categorisation model for longitudinal injury surveillance in elite rugby and cricket: intersport comparisons and inter-rater reliability of coding. Br J Sports Med 2017. doi: bjsports-2016-097040.

2 RFU. England Profession Rugby Injury Surveillance Project: 2015-2016 Season Report, 2017.

3 Holtzhausen LJ, Schwellnus MP, Jakoet I, et al. The incidence and nature of injuries in South African rugby players in the rugby Super 12 competition. S Afr Med J 2006:96:1260-5.

4 Gardner AJ, Iverson GL, Williams WH, et al. A systematic review and meta-analysis of concussion in rugby union. Sports Med 2014;44:1717-31.

5 WorldRugby. Concussion management: Recognise and Remove. http://playerwelfare. worldrugby.org/?documentid=112 (accessed 26 Sep 2017).

6 Moore IS, Ranson C, Mathema P. Injury risk in International Rugby Union: Three-year injury surveillance of one National team. Orthop I Sport Med 2015:3:2325967115596194.

7 Cross M, Kemp S, Smith A, et al. Professional Rugby Union players have a 60\% greater risk of time loss injury after concussion: a 2-season prospective study of clinical outcomes. Br J Sports Med 2016;50:926-31.

8 Nordström A, Nordström P, Ekstrand J. Sports-related concussion increases the risk of subsequent injury by about $50 \%$ in elite male football players. Br J Sports Med 2014:48:1447-50.

9 Brooks MA, Peterson K, Biese K, et al. Concussion increases odds of sustaining a lower extremity musculoskeletal injury after return to play among collegiate athletes. Am J Sports Med 2016:44:742-7.

10 Herman DC, Jones D, Harrison A, et al. Concussion may increase the risk of subsequent lower extremity musculoskeletal injury in collegiate athletes. Sports Med 2017;47:1003-10.

11 Fuller CW, Molloy MG, Bagate C, et al. Consensus statement on injury definitions and data collection procedures for studies of injuries in rugby union. Br J Sports Med 2007:41:328-31.

12 Rae K, Orchard J. The Orchard Sports Injury Classification System (OSICS) version 10 Clin J Sport Med 2007:17:201-4.

13 Efron B. The jackknife, the bootstrap and other resampling plans. Philadelphia, Pa: Society for Industrial and Applied Mathematics, 1982

14 der WSvan, Colbert SC, Varoquaux G. The NumPy Array: A structure for efficient numerical computation. Comput Sci Eng 2011;13:22-30.

15 Ullah S, Gabbett TJ, Finch CF. Statistical modelling for recurrent events: an application to sports injuries. Br J Sports Med 2014;48:1287-93.

16 Mahmood A, Ullah S, Finch CF. Application of survival models in sports injury prevention research: A systematic review. Br J Sports Med 2014;48:630.2-630.

17 Therneau T, Grambsch P. Modeling survival data: extending the cox model. New York: Spring-Verlag, 2013

18 Therneau T. Mixed effects Cox models. R Packag 2015

19 Fuller CW, Laborde F, Leather RJ, et al. International Rugby Board Rugby World Cup 2007 injury surveillance study. Br J Sports Med 2008:42:452-9.

20 Fuller CW, Sheerin K, Targett $S$, et al. International Rugby Board injury surveillance study. Br J Sports Med 2011;2013:1184-91.

21 Fuller CW, Taylor A, Kemp SP, et al. Rugby World Cup 2015: World Rugby injury surveillance study. Br J Sports Med 2017;51:51-7.

22 Ranson C, George J, Rafferty J, et al. Playing surface and UK professional rugby union injury risk. J Sports Sci.

23 Tucker R, Raftery M, Kemp S, et al. Risk factors for head injury events in professional rugby union: a video analysis of 464 head injury events to inform proposed injury prevention strategies. Br J Sports Med 2017;51:1152-7.

24 WorldRugby. World Rugby training and education: Coaching key factors.. http:// coaching.worldrugby.org/downloads/Coaching_Key_Factors_EN.pdf (accessed 26 Sep 2017).

25 WorldRugby. World Rugby concussion guidance. http://playerwelfare.worldrugby.org/? documentid=158 (accessed 26 Sep 2017)

26 Gabbett TJ. The training-injury prevention paradox: should athletes be training smarte and harder? Br J Sports Med 2016:50:273-80.

27 Gabbett TJ, Hulin BT, Blanch P, et al. High training workloads alone do not cause sports injuries: how you get there is the real issue. Br J Sports Med 2016.

28 Howell DR, Osternig LR, Chou LS. Return to activity after concussion affects dual-task gait balance control recovery. Med Sci Sports Exerc 2015;47:673-80.

29 Parker TM, Osternig LR, Lee HJ, et al. The effect of divided attention on gait stability following concussion. Clin Biomech 2005;20:389-95.

30 Parker TM, Osternig LR, VAN Donkelaar $\mathrm{P}$, et al. Gait stability following concussion. Med Sci Sports Exerc 2006;38:1032-40.

31 Schneider KJ, Meeuwisse WH, Nettel-Aguirre A, et al. Cervicovestibular rehabilitation in sport-related concussion: a randomised controlled trial. Br J Sports Med 2014:48:1294-8

32 McCrory P, Meeuwisse W, Dvořák J, et al. Consensus statement on concussion in sport-the $5^{\text {th }}$ international conference on concussion in sport held in Berlin, October 2016. Br J Sports Med 20172017;51. 